\title{
Análise do desempenho do retorno das ações ordinárias de empresas do setor do agronegócio em cenários econômicos adversos
}

\author{
Analysis of the performance of the return on common shares of companies \\ in the agribusiness sector in adverse economic scenarios
}

\section{José Odálio dos Santos}

Pontifícia Universidade Católica (PUC) - SP

email:j.odalio@pucsp.br

\section{Fernando Almeida Santos}

Pontifícia Universidade Católica (PUC) - SP

email:fernando@fernandoasantos.com.br

\section{Luis Antonio Volpato}

Pontifícia Universidade Católica (PUC) - SP

email:Ivolpato@pucsp.br

\section{Bruno Luis Volpato}

Pontifícia Universidade Católica (PUC) - SP

email:brunovolpato@gmail.com

\section{RESUMO}

O agronegócio brasileiro tem apresentado histórico de geração contínua de superávits comerciais. Para avaliar se empresas de referência do setor apresentaram resultados favoráveis e retornos anormais, mesmo em cenários de crise econômica, este artigo analisou os fatores determinantes e o desempenho do retorno das ações ordinárias. Foram utilizadas as metodologias de painel de dados e de estudos de eventos para o período de 2015 a 2020, que incluiu os cenários recessivos de 2015 e 2016 e 2020 com a deflagração da pandemia Covid-19. As variáveis independentes selecionadas, representam dados que capturam parcialmente o desempenho contábil, setorial e de conjuntura econômica do período analisado. Verificou-se uma correlação negativa entre o retorno das ações ordinárias e o PIB e o EMBI e $p$-values não significativos nos períodos ex-ante ex-post ao anúncio da pandemia, evidenciando que o desempenho de empresas do agronegócio tende a não ser afetado significativamente em cenários econômicos adversos.

Palavras-Chave: agronegócio; EBITDA; retorno das ações ordinárias

\section{ABSTRACT}

Brazilian agribusiness has shown a history of continuous generation of trade surpluses. In order to assess whether reference companies in the sector presented favorable results and abnormal returns, even in economic crisis scenarios, this article analyzed the determining factors and the performance of the return on common shares. Panel data and event studies methodologies were used for the period 2015 to 2020, which included the economic recession years of 2015 and 2016 and 2020 with the outbreak of the Covid-19 pandemic. The selected independent variables represent data that partially capture the accounting, sectorial and economic situation performance of the analyzed period. There was a negative correlation between the return on common shares and GDP and the EMBI and non-significant $p$-values in the ex-ante and ex-post periods to the pandemic announcement, showing that the performance of agribusiness companies tends not to be significantly affected in adverse economic scenarios.

Key-words: agribusiness; EBITDA; return of common shares 


\section{INTRODUÇÃO}

O agronegócio apresenta características das múltiplas atividades empresariais, como operações de compra, venda, contratação de serviços, gerenciamento de fornecedores e atendimento a clientes.

Conforme dados fornecidos pela Confederação da Agricultura e Pecuária do Brasil (CNA), observados na Figura 1, o valor bruto do faturamento no Brasil, em 2019 e 2020, foi gerado por produtos extraídos da plantação, principalmente a soja ( $\mathrm{R} \$ 175,6$ bilhões) que foi responsável por aproximadamente $\mathrm{R} \$ 1,00$ de cada $\mathrm{R} \$ 4,00$ da produção do setor. $\mathrm{Na}$ sequência, destacaram-se o milho ( $\mathrm{R} \$ 90,7$ bilhões), a cana-de açúcar ( $\mathrm{R} \$ 47,4$ bilhões), o café ( $\mathrm{R} \$ 28,6$ bilhões) e o algodão ( $\mathrm{R} \$ 20,5$ bilhões). Esses produtos foram os responsáveis principais da pauta de exportações brasileiras nesse período, representando aproximadamente $45 \%$ do valor das transações de comércio exterior.

Os sucessivos superávits comerciais do agronegócio brasileiro, desde 2008, têm mais que superado o déficit comercial dos demais setores da economia brasileira, e garantido sucessivos superávits à Balança Comercial Brasileira - ver Figura 2, com dados disponibilizados pela Confederação da Agricultura e Pecuária do Brasil (CNA).

Mesmo com a pandemia de Covid-19, que afetou a economia mundial, o agronegócio brasileiro venceu os desafios e vai encerrar o ano com crescimento de 9\% no Produto Interno Bruto (PIB), frente a 2019. A maior demanda interna e externa pelos vários produtos agrícolas e pecuários, a valorização dos preços e aumento de produção são fatores que contribuíram para o incremento significativo.

O setor agropecuário também foi destaque nas transações de comércio exterior entre janeiro e outubro de 2020. As exportações cresceram 5,7\% em receita nos 10 primeiros meses de 2020. Em volume a alta foi de $12 \%$ nesse período. Os cinco principais destinos foram: China, União Europeia, Estados Unidos, Japão e Coreia do Sul, que juntos representaram 63\% do total das exportações brasileiras em 2020.

Por tudo exposto, considerou-se como contribuição para o debate acadêmico, avaliar a significância estatística de um conjunto de variáveis independentes para explicar a variabilidade do retorno das ações ordinárias de duas empresas de referência do setor (BrasilAgro e SLC Agrícola), segundo a classificação da B3 (Brasil, Bolsa, Balcão), cujo faturamento é resultante principalmente da transformação dos produtos soja, algodão, milho, café e cana-de-açúcar. No grupo de variáveis selecionadas, $o$ interesse principal foi verificar a coerência do sinal do coeficiente na fórmula de regressão e a significância estatística de variáveis que, em parte, capturariam o desempenho do cenário econômico brasileiro: o Produto Interno Bruto (PIB) e o Índice

Figura 1 Valor Bruto do faturamento no Brasil em 2019 e 2020 (em R\$ bilhões)

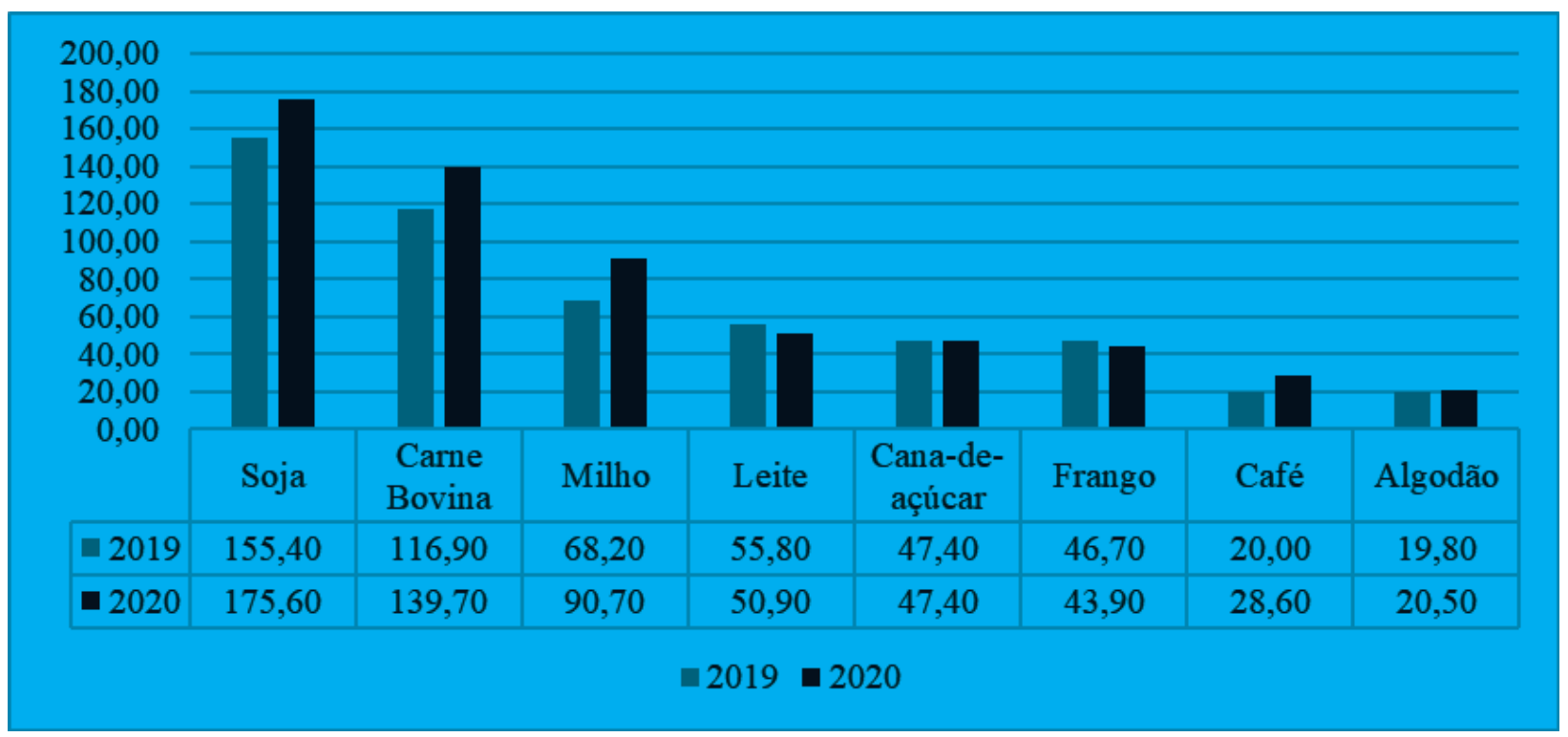

Fonte Confederação da Agricultura e Pecuária do Brasil (CNA), maio/2020. 
Figura 2 Saldo da Balança Comercial Brasileira de 2008 a 2019 (em US\$ bilhões)

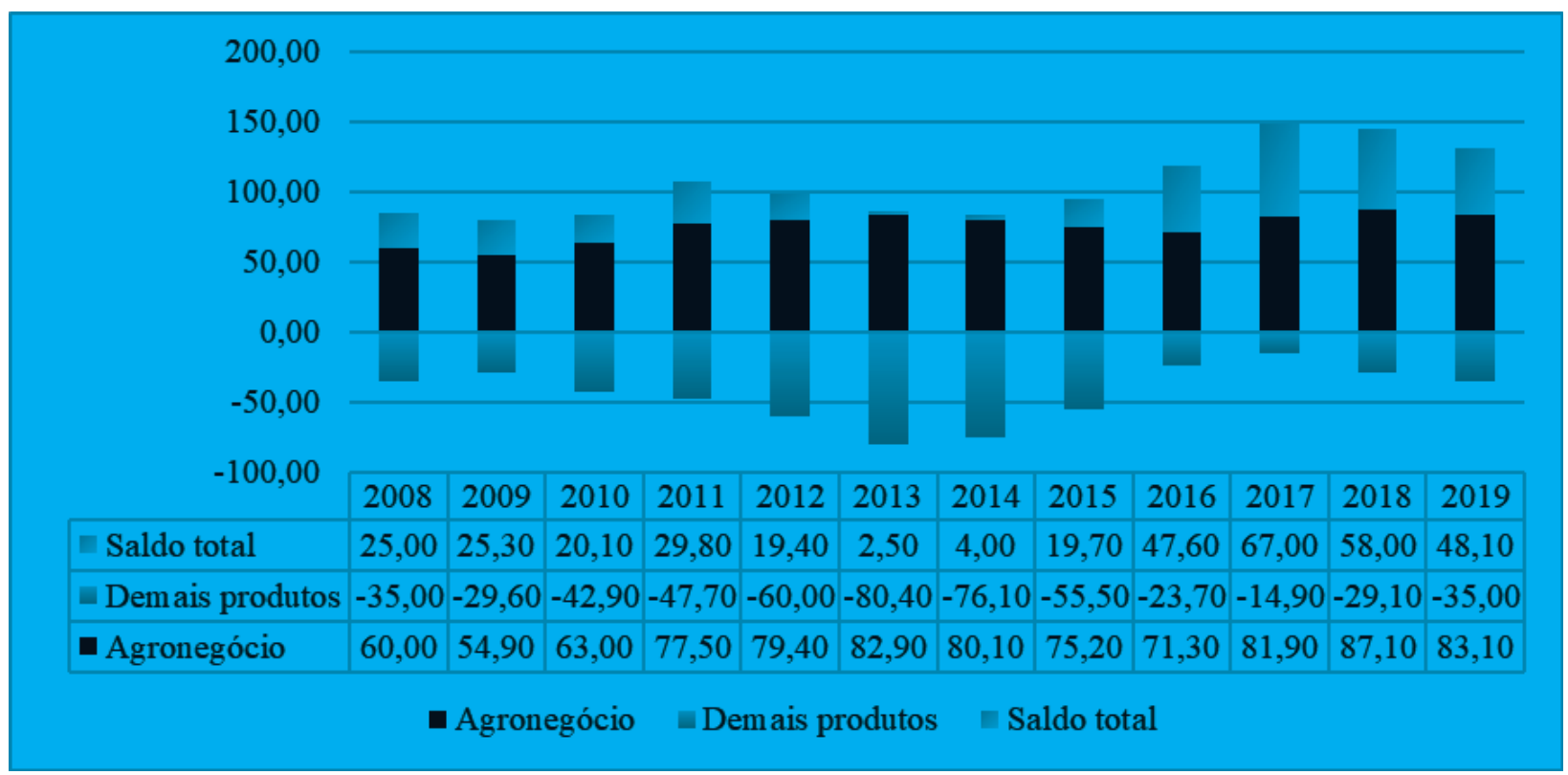

Fonte Instituto Brasileiro de Geografia e Estatística (IBGE) e Confederação da Agricultura e Pecuária do Brasil (CNA), maio/2020.

de Títulos de Mercados Emergentes (EMBI BR). Para dar sustentação a sensibilidade baixa do setor do agronegócio a ocorrência de eventos sistêmicos adversos, procedeu-se a verificação da ocorrência de retornos anormais estatisticamente significativos nos cenários de recessão de 2015 e 2020, respectivamente resultantes da crise política e econômica e da deflagração da pandemia Covid-19 pela Organização Mundial de Saúde (OMS).

Através dessa pesquisa procurou-se responder os seguintes problemas de pesquisa:

A variabilidade das variáveis explicativas selecionadas, de natureza contábil, setorial e econômica, contribui, de forma estatisticamente significativa, para explicar a variabilidade do retorno das ações ordinárias das empresas selecionadas;

O anúncio da deflagração da Pandemia Covid-19 contribuiu para a geração de retornos anormais estatisticamente significativos no período ex-post.

\section{FUNDAMENTAÇÃO TEÓRICA}

\subsection{Agronegócio}

Segundo Davis e Goldberg (1957), o agronegócio pode ser definido como a soma de todas as operações envolvidas na fabricação e distribuição de insumos agrícolas, operações de produção na fazenda e armazenamento, processamento e distribuição de commodities agrícolas.

Esse conceito surgiu a partir da integração da agricultura aos setores industriais de fornecimento de insumos, de um lado, e de processamento e distribuição da produção, de outro. Ele abrange todas as transformações associadas aos produtos agrícolas, desde a produção de insumos, passando pela unidade agrícola, processamento e distribuição até o consumidor final. O termo mostra uma acepção da qual participam também os agentes que produzem e coordenam o fluxo dos produtos, como os mercados, as entidades comerciais e as instituições financeiras (RUFINO, 1999; BURANELLO, 2017).

\subsection{Riscos do agronegócio}

O risco é um aspecto importante do negócio agrícola. As incertezas inerentes ao clima, produtividade, preços, políticas governamentais, mercados globais e outros fatores que afetam a agricultura podem causar grandes oscilações na renda agrícola. A gestão de riscos envolve a escolha de alternativas que reduzam os efeitos financeiros que podem resultar de tais incertezas. 
O agronegócio está exposto aos seguintes riscos específicos: riscos operacionais, de mercado, financeiros e institucionais (MOREIRA, 2009; USDA, 2020).

Os riscos operacionais são aqueles ligados ao processo produtivo e consequência da forma de gestão da empresa. O risco de produção deriva dos processos de crescimento natural incertos das culturas e da pecuária. O clima, as doenças, as pragas e outros fatores afetam a quantidade e a qualidade das mercadorias produzidas. Estes são os mais difíceis de prever e controlar os efeitos. Essas incertezas consistem em eventos físicos e biológicos que se traduzem em riscos climáticos e fitopatológicos que podem vir a prejudicar as condições de cultivo das culturas agrícolas e reduzir a produtividade das lavouras. Com relação ao clima, por exemplo, podem-se citar vários eventos como chuvas excessivas ou insuficientes, granizos, variações bruscas de temperatura etc. Quanto a forma de gestão, pode-se destacar as perdas no processo produtivo, decorrentes, por exemplo, de falhas no plantio e colheita - acidentes no trabalho, problemas mecânicos em maquinários, entre outros. Dentre as alternativas de mecanismos de proteção contra o risco agronômico, destaca-se o seguro rural, que ainda não é uma prática amplamente disseminada no mercado brasileiro devido ao alto custo.

Os riscos de mercado são associados à flutuação dos preços (decorrentes de variação na oferta e demanda de commodities) e outras variáveis econômicas que impactam, concomitantemente, os custos e as receitas dos negócios. Tais variáveis incluem, dentre outras, a taxa de juros, a taxa de câmbio e o crescimento da renda. Dentre as alternativas para mitigar a exposição aos riscos de mercado, destacam-se contratos futuros, opções, contratos a termo e swaps cambiais. Tais mecanismos atuam na fixação efetiva de preços (como nos mercados futuros e a termo) e no hedge cambial (como no swap). Segundo Nakao (2017), as estratégias de proteção de riscos, como a proteção por meio de derivativos, movimentam o mercado financeiro.

Os riscos financeiros estão relacionados à estrutura de capital e alavancagem que determinam a sensibilidade da dívida da empresa em relação às condições de mercado, ao desempenho operacional e à liquidez das empresas do setor. Esse risco surge quando a empresa agrícola toma dinheiro emprestado para financiar suas necessidades de capital de giro e capital permanente. $\mathrm{O}$ aumento das taxas de juros e a disponibilidade restrita de crédito também são aspectos do risco financeiro.

Os riscos institucionais são decorrentes de alterações nas legislações, políticas fiscais, tarifárias e de juros, restrições ambientais, normas sanitárias e práticas de organismos internacionais de regulação (como a Organização Mundial do Comércio), que podem impactar no agronegócio. Na prática, os riscos podem se manifestar como criação de novos tributos ou de leis ambientais restritivas, sanções de órgãos internacionais de regulação, represálias de países importadores, entre outros.

\subsection{Relação entre anúncio de informações relevantes e o preço da ação}

De acordo com a hipótese de eficiência de mercado, um mercado é eficiente se seus preços refletem completamente, tanto as informações disponíveis, como o anúncio de informações relevantes com conteúdo inesperado (FAMA, 1970, KUTTNER, 2001).

A Figura 3 mostra a diferença entre o mercado eficiente e o mercado ineficiente. Se o mercado for eficiente, o preço da ação será ajustado imediatamente ao anúncio da informação relevante - isto ocorrerá na data 0 , que representa o momento exato da divulgação da informação. No mercado ineficiente a informação não é absorvida na data 0 , ou seja, o preço da ação tende a atingir o novo nível de equilíbrio, nos próximos dias.

Figura 3 Reação do preço das ações ao anúncio de informações relevantes novas

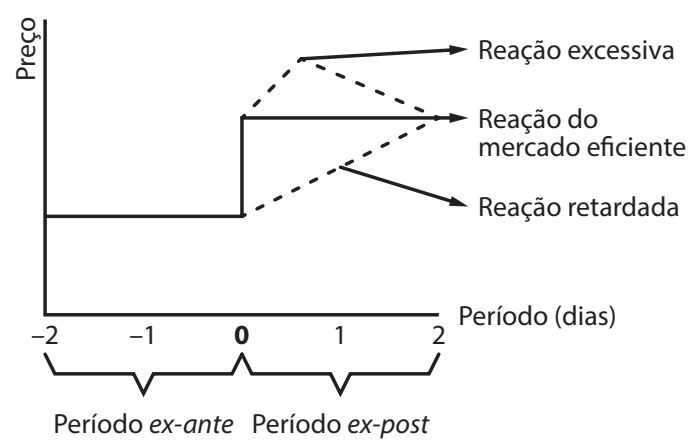

Fonte Adaptada de Ross, Westerfield e Jaffe et al (1995) 
Fama (1970) destaca três tipos de formas de eficiência de mercado: fraca, semi-forte e forte. Quando os dados históricos de mercado e os preços anteriores são totalmente refletidos pelo preço das ações, mas não podem prever o preço futuro, trata-se da forma de eficiência fraca. Caso os preços das ações se ajustem às novas informações publicamente disponíveis de forma rápida e imparcial, sem a possibilidade de obtenção de retorno excedente, tem-se a forma de eficiência semi-forte. $\mathrm{Na}$ forma de eficiência forte, $\mathrm{o}$ pressuposto é que todas as informações, públicas e privadas, são refletidas no preço das ações. Essa forma de eficiência de mercado não é possível onde existem barreiras legais para que as informações privadas se tornem públicas.

Para Kim e Verrecchia (1991), é questionável a existência de mercado eficiente perfeito, tendo em vista que os investidores são diversamente informados e diferem na precisão de suas informações pessoais anteriores. Indo mais além, poder-se-ia destacar que nem todos os investidores têm acesso as mesmas informações, princípios éticos, formação etc. Por isso, nem sempre analisam essas informações da mesma forma.

As informações divulgadas podem ser de natureza endógena, ou intrínseca a atividade operacional da empresa (ex. tecnologia, controle de qualidade, estratégia comercial, governança corporativa, desempenho contábil-financeiro etc.), e exógena, representando eventos sistêmicos que afetam o nível de atividade de todas as empresas no mercado (ex.: inflação, taxa de juros, câmbio, carga tributária, clima, doenças etc. (SANTOS, 2019).

Conforme Houlthousen e Verrechia (1990), o preço da ação apresentará reação significativa somente quando a informação divulgada for considerada relevante - subentende-se representativa da capacidade de geração de fluxos de caixa futuros e do risco do investimento.

A Tabela 1 apresenta pesquisas acadêmicas destacando o impacto de anúncio de informações relevantes sobre o preço das ações.

Especificamente para essa pesquisa, foi analisado o impacto do anúncio de informações relevantes

Tabela 1 Pesquisas abordando o impacto de anúncios sobre o preço das ações

\begin{tabular}{|c|c|}
\hline Autor & Relação encontrada após a divulgação de informações relevantes no mercado \\
\hline $\begin{array}{l}\text { Boyd, Hu e Jagannathan, } \\
\text { (2005) }\end{array}$ & $\begin{array}{l}\text { Surpresas na divulgação de notícias macroeconômicas afetam o preço das ações. A resposta das ações depende } \\
\text { se a economia está expandindo ou contraindo. Em média, o mercado de ações responde positivamente as } \\
\text { notícias do aumento de desemprego em expansões, e negativamente em contrações. }\end{array}$ \\
\hline Robitaille e Roush (2006) & $\begin{array}{l}\text { Surpresas na divulgação de dados agregados macroeconômicos americanos são capazes, em média, de afetar o } \\
\text { mercado de ações brasileiro. }\end{array}$ \\
\hline Wongsman (2009) & $\begin{array}{l}\text { Informações de política monetária estão significativamente associadas as oscilações no mercado de ações } \\
\text { brasileiro. }\end{array}$ \\
\hline Gonçalves e Eid (2011) & $\begin{array}{l}\text { Divulgação de decisões de política monetária brasileira sobre o mercado de ações, relacionadas a taxa de juros, } \\
\text { estariam associadas a quedas nos preços das ações de empresas brasileiras. Segundo este estudo, um aumento } \\
\text { não antecipado de } 1 \% \text { na meta SELIC levaria, em média, a uma queda de 1,3\% do Ibovespa. }\end{array}$ \\
\hline Birz e Lott (2011) & Notícias sobre o PIB e a taxa de desemprego afetam significativamente o retorno das ações. \\
\hline Bastos (2014) & $\begin{array}{l}\text { Anúncio de dados, tanto da conjuntura econômica brasileira como americana afetam conjuntamente, de forma } \\
\text { significativa, o mercado de ações brasileiro. }\end{array}$ \\
\hline Ashraf (2020) & $\begin{array}{l}\text { Anúncios de medidas de distanciamento social por agentes do governo apresentaram um efeito negativo sobre } \\
\text { o retorno de títulos no mercado de ações devido ao seu impacto adverso sobre a atividade econômica. }\end{array}$ \\
\hline $\begin{array}{l}\text { Khan, Zhao, Zhang, Yang e } \\
\text { Jahanger (2020) }\end{array}$ & $\begin{array}{l}\text { No curto prazo, o anúncio da transmissibilidade do Covid-19 influenciou negativamente a pontuação da Bolsa } \\
\text { de Valores de Xangai. A medida em que o governo chinês adotou medidas drástica para conter a propagação } \\
\text { da pandemia, os investidores recuperaram a confiança e, com isso, o desempenho da pontuação da carteira de } \\
\text { mercado melhorou nas janelas de estudo selecionadas. }\end{array}$ \\
\hline $\begin{array}{l}\text { Eldomiaty, Saeed, } \\
\text { Hammam e AboulSoud } \\
\text { (2020) }\end{array}$ & $\begin{array}{l}\text { Mudanças nas taxas de juros reais e nas taxas de inflação Granger causam mudanças siginificativas nos preços } \\
\text { das ações. }\end{array}$ \\
\hline
\end{tabular}

Fonte Elaborado pelos autores. 
relacionadas ao Produto Interno Bruto e a saúde pública, que contribuíram para a agravamento do cenário econômico. O evento 1 corresponde ao anúncio de informações relevantes no mercado nos dias 06 de julho de 2015 e 06 de janeiro de 2016. Na primeira data, foi anunciado o segundo semestre consecutivo de involução do Produto Interno Bruto (PIB). Na segunda data, foi anunciado o quarto semestre de desempenho negativo no nível de atividade econômica do Brasil, totalizando uma queda de $3,8 \%$ PIB em 2015 - trata-se da pior crise na economia brasileira antes do Covid-19. Contribuíram para esse cenário econômico adverso em 2015, o câmbio e juros elevados, a divulgação de escândalos de corrupção na maior empresa estatal do país (Petrobras) e o avanço da operação Lava Jato. O evento 2, corresponde ao anúncio da informação relevante no dia 11 de março de 2020, quando a Organização Mundial da Saúde (OMS) declarou a pandemia do Covid-19.

\section{METODOLOGIA DE PESQUISA}

Segue descrição sintética das empresas de referência do setor do agronegócio selecionadas para avaliação de resultados extraídos das metodologias de painel de dados e estudo de eventos. A BrasilAgro - Companhia Brasileira de Propriedades Agrícolas, fundada em 2005, obtém receitas principalmente resultantes do cultivo de soja, milho, sorgo e algodão,

Tabela 2 Variáveis de pesquisa

\begin{tabular}{|c|c|}
\hline $\begin{array}{l}\text { Variável } \\
\text { Dependente }\end{array}$ & Significado \\
\hline RAO & Retorno das ações ordinárias \\
\hline $\begin{array}{l}\text { Variáveis } \\
\text { Independentes }\end{array}$ & Significado \\
\hline EBITDA & $\begin{array}{l}\text { Earnings before interest, taxes, depreciation and amortization - value } \\
\text { Lucro antes dos juros, impostos, depreciação e amortização - valor }\end{array}$ \\
\hline ROIC & Retorno sobre o capital investido = Lucro operacional ajustado/Capital operacional investido - percentual \\
\hline IC-BR & $\begin{array}{l}\text { Índice de commodities - Brasil - Agropecuária. O IC-Br é construído com base nos preços das commodities agrícolas, me- } \\
\text { tálicas e energéticas convertidos para reais. Entre os três subgrupos que compõem o IC-Br, o de commodities agropecuá- } \\
\text { rias considera a variação do preço dos itens seguintes: carne de boi, carne de porco, algodão, óleo de soja, trigo, açúcar, } \\
\text { milho, arroz, café, suco de laranja e cacau. Quanto maior o IC-BR, maior o retorno obtido pelo investidor. }\end{array}$ \\
\hline TJCR & Taxa média de juros das operações de crédito com recursos direcionados - pessoas jurídicas \\
\hline IF & Importação de fertilizantes - em quantidade. \\
\hline EA & Exportações - setor: agricultura e pecuária - (FOB) \\
\hline PS & Preço da soja - valor \\
\hline PIB & Produto Interno Bruto do Brasil - percentual \\
\hline PC & Paridade R\$/US\$ - valor \\
\hline IPC & Índice de preços ao consumidor - percentual \\
\hline POR & Pessoas ocupadas com rendimento - quantidade \\
\hline EF & $\begin{array}{l}\text { Endividamento das famílias com o Sistema Financeiro Nacional em relação à renda acumulada dos últimos doze meses - } \\
\text { percentual }\end{array}$ \\
\hline $\mathrm{EMBI} \mathrm{Br}$ & $\begin{array}{l}\text { Emerging markets bond index - value } \\
\text { Índice de títulos de mercados emergentes Brasil - valor }\end{array}$ \\
\hline RI & Retorno da pontuação do Ibovespa \\
\hline
\end{tabular}

Fonte Elaborada pelos autores com dados extraídos dos sites do IPEADATA, Banco Central do Brasil, Finance Yahoo, Morningstar, BrasilAgro e SLC Agrícola 
além da cana-de-açúcar. A empresa possui 10 fazendas em 6 estados brasileiros e 1 fazenda no Paraguai com uma área total de 215.330 hectares de terras próprias e 53.735 hectares de terras arrendadas.

A SLC Agrícola, fundada em 1977, é uma das maiores produtoras mundiais de grãos e fibras, focada na produção de algodão, soja e milho. Foi uma das primeiras empresas do setor a ter ações negociadas em Bolsa de Valores no mundo, tornando-se uma referência no seu segmento. Com Matriz em Porto Alegre (RS), a Empresa possui 16 Unidades de Produção estrategicamente localizadas em 6 estados brasileiros que totalizaram 448.568 hectares no ano-safra 2019/20 - sendo 125.462 ha de algodão, 235.444 ha de soja, 82.392 ha de milho e 5.270 ha de outras culturas.

A Tabela 2 apresenta as variáveis de pesquisa selecionadas, prontas e/ou convertidas para periodicidade mensal.

Para justificar a escolha do EBITDA como variável independente e métrica de referência do desempenho contábil-financeiro das empresas do setor de agronegócio, destaca-se o fato de representar o lucro gerado na atividade operacional, isento das deduções de depreciação e amortização. Esse indicador é utilizado pela predominância das instituições financeiras de grande porte para avaliar se seus clientes apresentam histórico de geração de lucro operacional mais do que suficiente para a cobertura do valor do serviço da dívida (valor do financiamento e despesas financeiros).

Quanto a seleção do retorno das ações como variável dependente, partiu-se da premissa de representar a capacidade futura das empresas gerarem fluxos de caixa operacionais mais do que suficientes para atender as exigências de retornos dos detentores do capital patrimonial (dividendos e ganhos de capital), mesmo em cenários econômicos adversos.

\subsection{Metodologia de painel de dados}

A metodologia de painel de dados considera observações de $n$ entidades para dois ou mais períodos temporais. Segundo Duarte, Lamounier e Takamatsu (2007), essa metodologia pressupõe uma combinação entre dados de corte transversal e de séries temporais, permitindo o controle da heterogeneidade. Dentre as vantagens dessa metodologia, destaca-se o fato de tolerar maior quantidade de informações e maior variabilidade de dados.

Para Wooldridge (2017) e Gujarati e Porter (2011), o modelo geral para dados em painel é representado por:

$$
\mathrm{Y}=\beta_{\mathrm{o}}+\beta_{1} \mathrm{X}_{1}+\beta_{2} \mathrm{X}_{2}+\ldots \beta_{\mathrm{n}} \mathrm{X}_{\mathrm{n}}+\varepsilon
$$

Y: Variável dependente ou variável a ser predita ou valor de saída do modelo;

$\boldsymbol{\beta}_{\mathrm{o}}$ : Valor de saída quando os previsores apresentam os valores zerados - coeficiente angular ou parâmetro intercepto do modelo;

$\boldsymbol{\beta}_{1}, \boldsymbol{\beta}_{2} \ldots \boldsymbol{\beta}_{\mathrm{n}}$ : Coeficientes betas ou parâmetros de inclinação do modelo;

$\mathbf{X}_{1}, \mathbf{X}_{2} \ldots \mathbf{X}_{\mathbf{n}}$ : Variáveis independentes ou variáveis previsoras de Y;

$\varepsilon$ : Termo de erro ou variável que pode contemplar fatores não observados que afetariam a variável dependente $\mathrm{Y}$.

Para validar a participação das variáveis independentes selecionadas no modelo, foram confrontados os modelos e efeitos fixos e aleatórios a partir da aplicação do Teste de Hausman, que assume a hipótese nula pressupondo que os estimadores dos modelos de efeitos fixos e aleatórios não diferem substancialmente. Assumiu-se a condição de significância estatística para $p$-value de até 0,10 ou $10 \%$. Nesse caso, sempre que o $p$-value dos valores previsores for inferior ao nível de significância arbitrado $(0,10$ ou $10 \%$ ), assume-se a condição que a variável independente e estatisticamente significativa, contribuindo para explicação do comportamento da variabilidade da variável dependente. Quando o p-value for superior ao nível de significância arbitrado $(0,10$ ou $10 \%)$, as variáveis previsoras não contribuem, de forma estatisticamente significativa, para a explicação da variabilidade da variável dependente.

\subsection{Metodologia de estudo de eventos}

Campbell, Lo e Mackinley (1997) definem estudo de evento como o método pelo qual é possível medir o efeito de um evento econômico no valor de uma determinada empresa. Tal método é possível e 
eficaz em função da hipótese de que, em função da racionalidade do mercado, o efeito de um evento será refletido imediatamente nos preços dos ativos.

De acordo com Binder (1998), na prática o estudo de evento tem sido utilizado para duas principais razões:

(i) Testar a hipótese nula de que o mercado eficientemente incorpora informações; e,

(ii) Examinar o impacto de um determinado evento na riqueza dos acionistas de uma determinada empresa, mantendo a hipótese de mercado eficiente referente à informação pública.

As datas zero do evento 1 correspondem aos dias 06 de julho de 2015 e 06 de janeiro de 2016. Na primeira data, foi anunciado o segundo semestre consecutivo de involução do Produto Interno Bruto (PIB). Na segunda data, foi anunciado o quarto semestre consecutivo de desempenho negativo no nível de atividade econômica do Brasil, que, em termos consolidados, representou uma queda de 3,8\% do PIB do Brasil em 2015 - trata-se da pior crise na economia brasileira antes do Covid-19. Contribuíram para esse cenário econômico adverso em 2015, o câmbio e juros elevados, a divulgação de escândalos de corrupção na maior empresa estatal do país (Petrobras) e o avanço da operação Lava Jato.

Para o evento 2, a data zero considerada foi o dia 11 de março de 2020, quando a Organização Mundial da Saúde (OMS) declarou o Covid-19 como pandemia.

A janela do evento corresponde ao período t- 5 e $t+5$, ou seja, os cinco dias anteriores e posteriores a data de anúncio do evento (data 0 ).

O cálculo do retorno normal para as ações foi baseado em uma série de retornos observados antes da ocorrência dos eventos selecionados. Após a definição do que seriam estes retornos normais, procedeu-se a comparação com os retornos efetivamente observados, em busca da identificação de comportamentos anormais de retornos em períodos próximos ao evento anunciado.

A carteira referencial de mercado selecionada foi a da Bolsa de Valores de São Paulo.
Segue a fórmula sintética utilizada para calcular o retorno das ações ordinárias das empresas selecionadas:

$$
\mathbf{R}_{\mathrm{i}, \mathrm{t}}=\alpha_{\mathrm{i}}+\beta_{\mathrm{i}} \mathrm{R}_{\mathrm{m}, \mathrm{t}}+\varepsilon_{\mathrm{i}^{\prime} \mathrm{t}}
$$

$$
\begin{aligned}
& \mathbf{R}_{\mathrm{i}, \mathrm{t}}: \text { Retorno do ativo i no período } \mathrm{t} \\
& \alpha_{\mathrm{i}}: \text { Intercepto } \\
& \boldsymbol{\beta}_{\mathrm{i}}: \text { Inclinação } \\
& \mathbf{R}_{\mathrm{m}, \mathrm{t}}: \text { Retorno da carteira de mercado no período } \mathrm{t} \\
& \varepsilon_{\mathrm{i}, \mathrm{t}}: \text { Termo de erro }
\end{aligned}
$$

Para a estimação do retorno normal, do coeficiente alfa (intercepto) e do coeficiente beta (inclinação), considerou-se o período de 90 dias que antecedeu a janela do evento. Campbell, Lo e Mackinlay (1997) recomendam que a janela de estimação não seja a mesma da janela de evento para que os parâmetros

\begin{tabular}{|c|c|c|c|c|}
\hline$D_{-95}$ & $D_{-6}$ & $\mathrm{D}_{-5}$ & $\mathrm{D}_{+5}$ & $\mathrm{D}_{+6}$ \\
\hline & & \multicolumn{2}{|c|}{ Janela do evento } & $\begin{array}{l}\text { Pós janela } \\
\text { do evento }\end{array}$ \\
\hline
\end{tabular}
estimados do modelo não sejam influenciados.

A Tabela 3 ilustra, de maneira geral, as janelas elaboradas para cumprir o objetivo principal desta pesquisa.

Tabela 3 Janela de estimação e janela de evento

Fonte elaborada pelos autores

Segue a hipótese da pesquisa:

Ho: Não existe retorno anormal após a divulgação de informações relevantes

\section{RESULTADOS}

A seguir são apresentados os resultados e respectivas análises extraídos das metodologias de painel de dados e estudo de eventos. 


\subsection{Painel de dados - análise dos resultados}

Para a seleção do modelo de efeitos mais adequado (empilhado, fixo e aleatório), decidiu-se inicialmente descartar o modelo empilhado, uma vez tratar-se de estimador simples que se baseia no comportamento uniforme de todos os indivíduos ao longo do tempo e na homogeneidade das observações - desconsidera a heterogeneidade existente entre as unidades de corte transversal (ex. empresas, famílias, países, estados etc.). Segundo Gujarati e Porter (2011), o modelo de efeitos empilhados é considerado a maneira mais simples e ingênua de estimação, pois desconsidera as dimensões tempo e espaço, ou seja, a heterogeneidade das unidades de corte transversal.

A escolha entre o modelo de efeitos fixos e o modelo de efeitos aleatórios baseou-se na análise de existência de colinearidade entre as variáveis independentes na matriz de correlação e de autocorrelação serial de primeira ordem definida pelo índice Durbin-Watson. Para a análise de colinearidade, assumiu-se a premissa de validar variáveis independentes com grau de correlação positiva moderada de até 0,69 , conforme classificação de Devore (2006):
Tabela 4 Interpretação de intervalos de correlação

\begin{tabular}{l|l}
\hline \multicolumn{1}{c|}{ Intervalo de correlação } & \multicolumn{1}{c}{ Interpretação } \\
\hline 0,00 a 0,19 & Correlação positiva bem fraca \\
\hline 0,20 a 0,39 & Correção positiva fraca \\
\hline 0,40 a 0,69 & Correlação positiva moderada \\
\hline 0,70 a 0,89 & Correlação positiva forte \\
\hline 0,90 a 1,00 & Correlação positiva muito forte \\
\hline
\end{tabular}

Fonte Devore (2006)

Quanto a inexistência de autocorrelação serial de primeira ordem, considerou-se como pertinente a afirmação de Pindyck e Rubinfeld (2004), que nas séries temporais é muito difícil assumir que esta premissa seja atendida, pois na grande maioria das vezes as séries estão fortemente relacionadas com seus dados históricos. Por isso, arbitrou-se por considerar que valores de índices Durbin-Watson próximos de 2 atenderiam a condição de inexistência de autocorrelação serial de primeira ordem significativa.

Para a definição do modelo mais adequado (modelo de efeitos fixos ou modelo de efeitos aleatórios) para analisar a relação entre a variável dependente e as variáveis independentes, utilizou-se o índice de Hausman, que determina a escolha do modelo de efeitos fixos para $p$-values inferiores a 0,05 ou $5 \%$. Caso contrário ( $p$-values superiores a 0,05 ou $5 \%$ ), a escolha é do modelo de efeitos aleatórios (WOOLDRIDGE, 2017).

Tabela 5 Matriz de correlação

\begin{tabular}{|c|c|c|c|c|c|c|c|c|c|c|c|c|c|c|c|}
\hline CORRELAÇÃO & 1 & 2 & 3 & 4 & 5 & 6 & 7 & 8 & 9 & 10 & 11 & 12 & 13 & 14 & 15 \\
\hline 1 Retorno da ação &, 000 & 0,134 & 0,076 & $-0,423$ & $-0,043$ & $-0,138$ & $-0,462$ & 0,067 & $-0,121$ & 0,378 & 0,215 & $-0,014$ & 0,412 & $-0,122$ & $-0,409$ \\
\hline 2 Ebitda & 134 & 1,000 & $-0,099$ & $-0,097$ & $-0,135$ & $-0,117$ & $-0,162$ & 0,073 & $-0,031$ & 0,106 & $-0,004$ & 0,111 & 0,215 & 0,038 & $-0,129$ \\
\hline 3 EMBI BR & 0,076 & $-0,099$ & 1,000 & 0,175 & 0,179 & 0,000 & 0,175 & $-0,397$ & 0,333 & $-0,052$ & $-0,459$ & 0,036 & 0,073 & $-0,097$ & 0,540 \\
\hline 4 Endividamento das famílias & $-0,423$ & $-0,097$ & 0,175 & 1,000 & 0,117 & 0,240 & 0,614 & $-0,189$ & 0,221 & $-0,589$ & $-0,218$ & 0,199 & $-0,619$ & 0,195 & 0,583 \\
\hline 5 Exportação do agronegócio & $-0,043$ & $-0,135$ & 0,179 & 0,117 & 1,000 & $-0,105$ & $-0,012$ & $-0,223$ & $-0,154$ & $-0,233$ & $-0,166$ & 0,042 & $-0,255$ & $-0,055$ & 0,224 \\
\hline 6 Importação de fertilizantes & $-0,138$ & $-0,117$ & 0,000 & 0,240 & $-0,105$ & 1,000 & 0,136 & $-0,064$ & 0,095 & $-0,172$ & $-0,083$ & 0,003 & $-0,164$ & $-0,098$ & 0,143 \\
\hline 7 Índice de commo & $-0,462$ & $-0,162$ & 0,175 & 0,614 & $-0,012$ & 0,136 & 1,000 & 0,033 & 0,254 & $-0,536$ & $-0,071$ & 0,092 & $-0,464$ & 0,055 & 0,656 \\
\hline $8 \quad I P C$ & 0,067 & 0,073 & $-0,397$ & $-0,189$ & $-0,223$ & $-0,064$ & 0,033 & 1,000 & $-0,021$ & 0,097 & 0,436 & $-0,069$ & 0,086 & 0,055 & $-0,209$ \\
\hline 9 Preço da soja & $-0,121$ & $-0,031$ & 0,333 & 0,221 & $-0,154$ & 0,095 & 0,254 & $-0,021$ & 1,000 & $-0,007$ & $-0,287$ & 0,023 & 0,023 & 0,093 & 0,306 \\
\hline $\begin{array}{l}10 \text { Pessoas ocur } \\
\text { mento }\end{array}$ & 0,378 & 0,106 & $-0,052$ & $-0,589$ & $-0,233$ & $-0,172$ & $-0,536$ & 0,097 & $-0,007$ & 1,000 & $-0,023$ & $-0,069$ & 0,630 & 0,369 & $-0,493$ \\
\hline 11 Retorno Ibovespa & 215 & $-0,004$ & $-0,459$ & $-0,218$ & $-0,166$ & $-0,083$ & $-0,071$ & 0,436 & $-0,287$ & $-0,023$ & 1,000 & $-0,041$ & 0,042 & $-0,158$ & $-0,339$ \\
\hline $12 \mathrm{ROIC}$ & $-0,014$ & 0,111 & 0,036 & 0,199 & 0,042 & 0,003 & 0,092 & $-0,069$ & 0,023 & $-0,069$ & $-0,041$ & 1,000 & 124 & 0,063 & 0,075 \\
\hline $\begin{array}{l}13 \text { Taxa de } \\
\text { crédito }\end{array}$ & 0,412 & 0,215 & 0,073 & $-0,519$ & $-0,255$ & $-0,164$ & $-0,464$ & 0,086 & 0,023 & 0,630 & 0,042 & $-0,124$ & 1,000 & $-0,121$ & $-0,443$ \\
\hline $14 \mathrm{PIB}$ & $-0,122$ & 0,038 & $-0,097$ & 0,195 & $-0,055$ & $-0,098$ & 0,055 & 0,055 & 0,093 & 0,369 & $-0,158$ & 0,063 & $-0,121$ & 1,000 & $-0,055$ \\
\hline 15 Paridade R\$/US\$ & $-0,409$ & $-0,129$ & 0,540 & 0,583 & 0,224 & 0,143 & 0,656 & $-0,209$ & 0,306 & $-0,493$ & $-0,339$ & 0,075 & $-0,443$ & $-0,055$ & 1,000 \\
\hline
\end{tabular}

Fonte Dados da pesquisa. 


\subsubsection{Análise de existência de multicolinearidade}

A matriz de correlação (Tabela 5)não apresentou graus de correlação positiva forte entre as variáveis independentes. Com isso, considerou-se a inclusão de todas a variáveis independentes no modelo selecionado.

\subsubsection{Análise de existência de autocorrelação serial de primeira ordem}

O índice de Durbin-Watson foi de 2,20 para o modelo considerado adequado (Modelo de Efeitos Fixos). Com isso, assumiu a condição de existência de autocorrelação não significativa entre os valores correntes mensais das variáveis independentes e seus valores mensais imediatamente anteriores.

Tabela 6 Teste Durbin-Watson

$$
\text { F-statistic }
$$
4.56 2.20

Fonte Elaborada pelos autores

\subsubsection{Modelo de efeitos adequado}

A seleção do modelo mais adequado foi definida pelo Teste de Hausman, que considera a validação da escolha do modelo de efeitos fixos sempre que o $p$-value for inferior a 0,05 ou $5 \%$. Caso contrário, a recomendação é para a escolha do modelo de efeitos aleatórios.

Tabela 7 Teste de Hausman

Correlated Random Effects - Hausman Test

Equation: Untitled

\begin{tabular}{|c|c|c|c|}
\hline \multicolumn{2}{|c|}{ Test period random effects } & \multicolumn{2}{l|}{ Phi-Sq. } \\
\hline Test Summary & $\begin{array}{c}\text { Chatistic } \\
\text { Chi-Sq. d.f. }\end{array}$ & P-value \\
\hline Period random & 0.00 & 5 & $0.00^{*}$ \\
\hline
\end{tabular}

* Hausman statistic set to zero.

Fonte Dados da pesquisa.
O p-value do Teste de Hausman foi 0.00, validando a escolha do Modelo de Efeitos Fixos como mais adequado para os fins da pesquisa.

A Tabela 8 apresenta o Modelo de Efeitos Fixos gerado pelo Software E-views 11.

Na Tabela 8, pode-se observar que a predominância das variáveis independentes apresentou relação de coerência com o comportamento, ou variabilidade do retorno das ações ordinárias da BrasilAgro e SLC Agrícola, embora o efeito não seja estatisticamente significativo. As variáveis estatisticamente significativas que apresentaram sinal de coerência foram POR ( $p$-value 0,03$)$ e RI ( $p$-value $0,03)$. Intuitivamente e, em parte, poder-se-ia admitir essa relação positiva considerando que o aumento da variabilidade do retorno da pontuação da carteira de mercado estaria relacionado com o aumento do nível de atividade econômica e de geração de lucro operacional e de pessoas ocupadas com rendimento. As variáveis IC-BR ( $p$-value 0,07$)$ e PIB ( $p$-value 0,00$)$, classificadas como estatisticamente significativas, apresentaram coeficientes com sinais intuitivamente contrários ao esperado, porém possíveis de questionamento, quando se considera a natureza cíclica dos principais produtos da carteira do agronegócio. Em parte, poder-se-ia argumentar que a relação negativa está relacionada a natureza não-cíclica e alta rotatividade dos produtos (ex. soja, trigo, milho, café, arroz etc.), mesmo em cenários econômicos adversos. Nesse contexto, as empresas não são afetadas significativamente nos seus níveis de atividades, mantendo capacidade de geração interna de caixa e de lucro operacional (EBITDA).

A Tabela 9, apresenta as demais variáveis independentes do Modelo de Efeitos Fixos, classificadas como não estatisticamente significativas, com os sinais dos coeficientes (efetivo e esperado).

A predominância das variáveis apresenta sinal compatível com o esperado para explicar a variabilidade do retorno das ações ordinárias das empresas selecionadas. Já as variáveis independentes consideradas como foco principal da pesquisa (PIB e EMBI $\mathrm{BR}$ ) apresentaram sinais contrários ao esperado, em parte, dando sustentação a classificação de natureza não-cíclica e alta rotatividade do setor do agronegócio, possibilitando as empresas bem administradas do setor apresentar desempenho favorável, mesmo em 
Tabela 8 Modelo de efeitos fixos

\begin{tabular}{|c|c|c|c|c|}
\hline \multicolumn{5}{|c|}{ Dependent Variable: RETORNO_DAS_ACOES } \\
\hline \multicolumn{5}{|c|}{ Method: Panel EGLS (Two-way random effects) } \\
\hline \multicolumn{5}{|c|}{ Date: 01/04/21 Time: 14:34 } \\
\hline \multicolumn{5}{|c|}{ Sample: 2015M01 2020M09 } \\
\hline \multicolumn{5}{|c|}{ Periods included: 69} \\
\hline \multicolumn{5}{|c|}{ Total panel (balanced) observations: 138} \\
\hline \multicolumn{5}{|c|}{ Wansbeek and Kapteyn estimator of component variances } \\
\hline Variable & Coefficient & Std. Error & t-Statistic & Prob. \\
\hline EBITDA & 0.013374 & 0.034584 & 0.386699 & 0.6997 \\
\hline $\mathrm{EMBI}$ & 0.053991 & 0.055808 & 0.967439 & 0.3353 \\
\hline EF & 1.145812 & 0.847098 & 1.352633 & 0.1787 \\
\hline EA & 0.017913 & 0.012925 & 1.385887 & 0.1684 \\
\hline IF & -0.028041 & 0.022796 & -1.230076 & 0.2211 \\
\hline IC BR & -0.227419 & 0.125996 & -1.804977 & 0.0736 \\
\hline IPC & 0.000143 & 0.000924 & 0.154343 & 0.8776 \\
\hline PS & 0.096339 & 0.086528 & 1.113387 & 0.2678 \\
\hline POR & 1.499300 & 0.698041 & 2.147867 & 0.0337 \\
\hline $\mathrm{Rl}$ & 0.160389 & 0.071801 & 2.233795 & 0.0273 \\
\hline ROIC & 0.071761 & 0.114869 & 0.624713 & 0.5333 \\
\hline TJCR & 0.067992 & 0.082050 & 0.828667 & 0.4089 \\
\hline PIB & -0.646567 & 0.228005 & -2.835753 & 0.0054 \\
\hline PC & -0.215346 & 0.143463 & -1.501060 & 0.1360 \\
\hline
\end{tabular}

Fonte Dados da pesquisa.

Tabela 9 Sinais efetivos e esperados dos coeficientes do modelo de efeitos aleatórios

\begin{tabular}{l|c|c}
\hline \multicolumn{1}{c|}{ Variable } & Coefficient sign & $\begin{array}{c}\text { Expected } \\
\text { coeficiente sign }\end{array}$ \\
\hline EBITDA & + & + \\
\hline EMBI BR & + & - \\
\hline EF & + & - \\
\hline EA & + & + \\
\hline IF & + & - \\
\hline IPC & + & + \\
\hline PS & + & + \\
\hline ROIC & + & - \\
\hline TJCR & - & + \\
\hline PIB & - & + \\
\hline PC & & + \\
\hline
\end{tabular}

Fonte Dados da pesquisa. cenários econômicos adversos, como na recessão de 2015 e na pandemia do Covid-19.

O próximo subitem aborda o comportamento do retorno das ações ordinárias das empresas selecionadas diante da divulgação da queda brusca do PIB em 2015 e da deflagração da pandemia do Covid-19 em 2020.

\subsection{Estudo de eventos - análise dos resultados}

Para dar sustentação para os resultados favoráveis do setor do agronegócio, mesmo em cenários econômicos adversos, procedeu-se a análise da variabilidade do retorno das ações ordinárias das empresas selecionadas, imediatamente após a divulgação, no mercado, de informações relacionadas ao agravamento do cenário econômico brasileiro.

As datas zero do evento 1 correspondem ao anúncio de informações relevantes no mercado nos dias 06 de julho de 2015 e 06 de janeiro de 2016. Na 
primeira data, foi anunciado o segundo semestre consecutivo de involução do Produto Interno Bruto (PIB). Na segunda data, foi anunciado o quarto semestre de desempenho negativo no nível de atividade econômica do Brasil, totalizando uma queda de 3,8\% PIB em 2015 - trata-se da pior crise na economia brasileira antes do Covid-19. Contribuíram para esse cenário econômico adverso em 2015, o câmbio e juros elevados, a divulgação de escândalos de corrupção na maior empresa estatal do país (Petrobras) e o avanço da operação Lava Jato.

A data zero do evento 2, corresponde ao anúncio da informação relevante no dia 11 de março de 2020, quando a Organização Mundial da Saúde (OMS) declarou a pandemia do Covid-19.

\subsubsection{Análise dos resultados - BrasilAgro e SLC Agrícola}

As Tabelas 10, 11 e 12 apresentam o retorno anormal, o retorno anormal acumulado e os $p$-values dos cinco dias anteriores e posteriores a divulgação das informações relevantes relacionadas ao PIB negativo e deflagração da pandemia Covid-19 pela OMS.

Constatou-se que todos os $p$-values dos períodos ex-ante e ex-post foram superiores a 0,05 , indicando a não rejeição da hipótese nula, que considera efeito não significativo da informação divulgada no comportamento do retorno das ações ordinárias das empresas selecionadas.

Todavia, o que se observa é a acumulação de retornos anormais negativos, ou seja, destruição parcial da riqueza dos investidores. Trata-se de comportamento admissível, por considerar o anúncio de informações negativas relacionadas a redução relevante no nível de atividade econômica, ou recessão, tanto no período histórico (recessão econômica de 2015) como no período ex-post deflagração da pandemia Covid-19. Especificamente para o setor do agronegócio, caracterizado como de atividade operacional não-cíclica e de alta rotatividade, o impacto, embora negativo, não foi estatisticamente significativo, não comprometeu, de forma estatisticamente significativa, a capacidade de geração interna de recursos da empresa. Os dados da Tabela 10 atestam o desempenho favorável das empresas selecionadas em termos de geração de EBITDA e do retorno das ações ordinárias nos cenários econômicos adversos de 2015 e 2020.

\section{CONSIDERAÇÕES FINAIS}

O fator determinante que motivou o desenvolvimento dessa pesquisa foi desempenho favorável histórico do setor do agronegócio, mesmo em cenários econômicos adversos, como na recessão econômica de 2015 e no cenário da pandemia do Covid-19 em 2020.

Por isso, considerou-se oportuna a ocasião para avaliar a significância estatística de um conjunto de variáveis independentes para explicar a variabilidade do retorno das ações ordinárias de duas empresas de referência do setor (BrasilAgro e SLC Agrícola), segundo a classificação da B3 (Brasil, Bolsa, Balcão), cujo faturamento é resultante principalmente da transformação dos produtos soja, algodão, milho, café e cana-de-açúcar. No grupo de variáveis selecionadas, o interesse principal foi verificar a coerência do sinal do coeficiente na fórmula de regressão e a significância estatística de variáveis que, em parte, capturariam o desempenho do cenário econômico brasileiro: o Produto Interno Bruto (PIB) e o Índice de Títulos de Mercados Emergentes (EMBI Br). Para dar sustentação a sensibilidade baixa do nível de atividades de empresas de referência do setor do agronegócio a ocorrência de eventos sistêmicos adversos, procedeu-se a verificação da ocorrência de retornos anormais estatisticamente significativos nos cenários de recessão de 2015 e 2020, respectivamente resultantes da crise política e econômica e da deflagração da pandemia Covid-19 pela Organização Mundial de Saúde (OMS).

Para isso, foram utilizadas as metodologias de painel de dados e de estudos de eventos para avaliação da significância estatística e coerência do sinal das variáveis independentes objeto principal do estudo PIB e EMBI BR no modelo de regressão; e para a verificação de geração de retornos anormais imediatamente ao anúncio no mercado de informações relevantes com conotação negativa no cenário econômico, como a recessão severa ocorrida em 2015 e a deflagração da pandemia do Covid-19, que deteriorou o nível de atividade econômica mundial em 2020. 
Tabela 10 Retorno anormal (RA) e retorno acumulado anormal (RAA) - Evento 1 - 06 de julho de 2015

\begin{tabular}{r|c|c|c|c|c|c|c|c}
\cline { 2 - 9 } & \multicolumn{4}{|c|}{ BrasilAgro } & \multicolumn{3}{c}{ SLC Agrícola } \\
\cline { 2 - 9 } & $\mathbf{R A}$ & $\mathbf{R A A}$ & $\boldsymbol{p}$-value RA & $\boldsymbol{p}$-value RAA & RA & RAA & $\boldsymbol{p}$-value RA & $\boldsymbol{p}$-value RAA \\
\hline-5 & 0,00137 & 0,00137 & 0,46439 & 0,46439 & $-0,03040$ & $-0,00635$ & 0,66200 & 0,66200 \\
\hline-4 & $-0,00324$ & $-0,00186$ & 0,55961 & 0,53440 & 0,01526 & $-0,01085$ & 0,58288 & 0,69316 \\
\hline-3 & $-0,00154$ & $-0,00341$ & 0,52326 & 0,55130 & 0,02109 & $-0,00520$ & 0,41507 & 0,57832 \\
\hline-2 & $-0,00208$ & $-0,00549$ & 0,52722 & 0,57145 & $-0,02726$ & $-0,01171$ & 0,58439 & 0,64939 \\
\hline-1 & $-0,00038$ & $-0,00587$ & 0,50450 & 0,56841 & 0,00179 & $-0,01052$ & 0,48609 & 0,62114 \\
\hline $\mathbf{0}$ & $-\mathbf{0 , 0 1 6 9 1}$ & $-\mathbf{0 , 0 2 2 7 8}$ & $\mathbf{0 , 6 7 4 8 9}$ & $\mathbf{0 , 7 2 9 3 4}$ & $\mathbf{0 , 0 0 1 3 4}$ & $-\mathbf{0 , 0 1 8 2 2}$ & $\mathbf{0 , 5 8 1 6 8}$ & $\mathbf{0 , 6 8 7 1 4}$ \\
\hline 1 & $-0,00502$ & $-0,02780$ & 0,54959 & 0,75504 & $-0,01441$ & $-0,02721$ & 0,58805 & 0,74971 \\
\hline 2 & $-0,00775$ & $-0,03555$ & 0,57141 & 0,79552 & $-0,01668$ & $-0,01754$ & 0,41132 & 0,65773 \\
\hline 3 & 0,01995 & $-0,01560$ & 0,33116 & 0,63361 & 0,06016 & $-0,03847$ & 0,67620 & 0,79958 \\
\hline 4 & $-0,02202$ & $-0,03762$ & 0,67626 & 0,78266 & $-0,04510$ & $-0,04230$ & 0,53164 & 0,80965 \\
\hline 5 & 0,01211 & $-0,02551$ & 0,40521 & 0,69331 & $-0,00330$ & $-0,06164$ & 0,64884 & 0,88839 \\
\hline
\end{tabular}

Fonte Dados da pesquisa.

Tabela 11 Retorno anormal (RA) e retorno acumulado anormal (RAA) - Evento 1 - 06 de janeiro de 2016

\begin{tabular}{r|c|c|c|c|c|c|c|c}
\cline { 2 - 9 } & \multicolumn{4}{|c|}{ BrasilAgro } & \multicolumn{4}{c}{ SLC Agrícola } \\
\cline { 2 - 9 } & RA & RAA & $\boldsymbol{p}$-value RA & $\boldsymbol{p}$-value RAA & RA & RAA & $\boldsymbol{p}$-value RA & $\boldsymbol{p}$-value RAA \\
\hline-5 & $-0,00635$ & $-0,00635$ & 0,66200 & 0,66200 & 0,01024 & 0,01024 & 0,01024 & 0,33247 \\
\hline-4 & $-0,00450$ & $-0,01085$ & 0,58288 & 0,69316 & $-0,00803$ & 0,00221 & $-0,00803$ & 0,47363 \\
\hline-3 & 0,00565 & $-0,00520$ & 0,41507 & 0,57832 & 0,00491 & 0,00712 & 0,00491 & 0,43097 \\
\hline-2 & $-0,00650$ & $-0,01171$ & 0,58439 & 0,64939 & $-0,01157$ & $-0,00445$ & $-0,01157$ & 0,53733 \\
\hline-1 & 0,00119 & $-0,01052$ & 0,48609 & 0,62114 & $-0,01720$ & $-0,02165$ & $-0,01720$ & 0,65840 \\
\hline $\mathbf{0}$ & $-\mathbf{0 , 0 0 7 7 0}$ & $-\mathbf{0 , 0 1 8 2 2}$ & $\mathbf{0 , 5 8 1 6 8}$ & $\mathbf{0 , 6 8 7 1 4}$ & $\mathbf{0 , 0 3 9 0 3}$ & $\mathbf{0 , 0 1 7 3 9}$ & $\mathbf{0 , 0 3 9 0 3}$ & $\mathbf{0 , 3 8 2 3 9}$ \\
\hline 1 & $-0,00899$ & $-0,02721$ & 0,58805 & 0,74971 & $-0,00058$ & 0,01681 & $-0,00058$ & 0,39455 \\
\hline 2 & 0,00968 & $-0,01754$ & 0,41132 & 0,65773 & $-0,00839$ & 0,00842 & $-0,00839$ & 0,45011 \\
\hline 3 & $-0,02093$ & $-0,03847$ & 0,67620 & 0,79958 & 0,00009 & 0,00851 & 0,00009 & 0,45245 \\
\hline 4 & $-0,00383$ & $-0,04230$ & 0,53164 & 0,80965 & 0,00822 & 0,01673 & 0,00822 & 0,41183 \\
\hline 5 & $-0,01934$ & $-0,06164$ & 0,64884 & 0,88839 & 0,00145 & 0,01818 & 0,00145 & 0,40870 \\
\hline
\end{tabular}

Fonte Dados da pesquisa.

Tabela 12 Retorno anormal (RA) e retorno acumulado anormal (RAA) - Evento 2 - 11 de março de 2020

\begin{tabular}{r|c|c|c|c|c|c|c|c}
\cline { 2 - 9 } & \multicolumn{4}{|c|}{ BrasilAgro } & \multicolumn{4}{c}{ SLC Agrícola } \\
\cline { 2 - 10 } & $\mathbf{R A}$ & $\mathbf{R A A}$ & $\boldsymbol{p}$-value RA & $\boldsymbol{p}$-value RAA & RA & RAA & $\boldsymbol{p}$-value RA & $\boldsymbol{p}$-value RAA \\
\hline-5 & 0,03687 & 0,03687 & 0,01947 & 0,01947 & 0,05013 & 0,05013 & 0,03143 & 0,03143 \\
\hline-4 & $-0,02775$ & 0,00912 & 0,86364 & 0,35926 & $-0,00594$ & 0,04418 & 0,56184 & 0,12360 \\
\hline-3 & $-0,03675$ & $-0,02763$ & 0,87768 & 0,80914 & $-0,03000$ & 0,01418 & 0,73544 & 0,38304 \\
\hline-2 & $-0,06037$ & $-0,08800$ & 0,95002 & 0,99176 & $-0,06567$ & $-0,05149$ & 0,88214 & 0,82374 \\
\hline-1 & 0,05921 & $-0,02879$ & 0,09081 & 0,74200 & 0,04812 & $-0,00338$ & 0,23599 & 0,52012 \\
\hline $\mathbf{0}$ & $-\mathbf{0 , 0 6 8 9 4}$ & $-\mathbf{0 , 0 9 7 7 3}$ & $\mathbf{0 , 9 2 1 7 9}$ & $\mathbf{0 , 9 7 7 7 4}$ & $-\mathbf{0 , 0 5 5 7 2}$ & $-\mathbf{0 , 0 5 9 0 9}$ & $\mathbf{0 , 7 7 6 0 5}$ & $\mathbf{0 , 7 8 9 5 6}$ \\
\hline 1 & $-0,08917$ & $-0,18691$ & 0,95414 & 0,99980 & $-0,04963$ & $-0,10872$ & 0,73299 & 0,91345 \\
\hline 2 & 0,06719 & $-0,11972$ & 0,13039 & 0,97744 & 0,08525 & $-0,02347$ & 0,17223 & 0,60269 \\
\hline 3 & $-0,07679$ & $-0,19650$ & 0,88228 & 0,99880 & $-0,08615$ & $-0,10962$ & 0,81112 & 0,86914 \\
\hline 4 & $-0,00196$ & $-0,19846$ & 0,51116 & 0,99770 & 0,08102 & $-0,02860$ & 0,22173 & 0,60664 \\
\hline 5 & $-0,05005$ & $-0,24852$ & 0,75487 & 0,99969 & $-0,05017$ & $-0,07877$ & 0,67659 & 0,76406 \\
\hline
\end{tabular}

Fonte Dados da pesquisa. 
Tabela 13 EBITDA, retorno das ações ordinárias e desempenho do PIB brasileiro em 2015 e 2020

\begin{tabular}{l|c|c|c|c}
\hline \multirow{2}{*}{\multicolumn{1}{c|}{ Dados }} & \multicolumn{2}{c|}{2015} & \multicolumn{2}{c}{2020} \\
\cline { 2 - 5 } & BrasilAgro & SLC Agrícola & BrasilAgro & SLC Agrícola \\
\hline EBITDA (Mil) & 338.743 & 392.301 & 224.533 & $545.795^{* 1}$ \\
\hline Retorno das ações ordinárias & $11,90 \%$ & $9,07 \%$ & $12,22 \%$ & $12,25 \%$ \\
\hline Desempenho do PIB & \multicolumn{2}{|c|}{$-3,80 \%$} & $-4,50 \%$ \\
\hline
\end{tabular}

*1 Lucro operacional acumulado no período de janeiro a setembro de 2020.

*22 Estimativa do Banco Mundial divulgada em 05 de janeiro de 2021.

Fonte Elaborada pelos autores

Os resultados mostraram uma relação contrária entre o comportamento da variabilidade do retorno das ações ordinárias e o comportamento da variabilidade do PIB e do EMBI Br. Em parte, poder-se-ia argumentar que a relação negativa está relacionada a natureza não-cíclica e alta rotatividade dos produtos (ex. soja, trigo, milho, café, arroz etc.), mesmo em cenários econômicos adversos. Nesse contexto, as empresas não são afetadas significativamente nos seus níveis de atividades, mantendo capacidade de geração interna de caixa e de lucro operacional (EBITDA).

\section{REFERÊNCIAS}

ASHRAF, B. N. Economic impact of government interventions during the COVID-19 pandemic: International evidence from financial markets. Journal of Behavioral and Experimental Finance, v. 27, September 2020. https://doi.org/10.1016/j. jbef.2020.100371

BASTOS, E. T. S. P. Impactos de Surpresas Macroeconômicas sobre o Mercado Brasileiro de Ações (Dissertação de Mestrado). Insper Instituto de Ensino e Pesquisa, São Paulo, SP, Brasil. 2014.

BINDER, J. J. The event study methodology since 1969. Review of Quantitative Finance and Accounting, v. 11, Issue 2. 1998.

BIRZ, G.; LOTT, J. R. The effect of macroeconomic news on stock returns: New evidence from newspaper coverage. Journal of Banking \& Finance. 2011. https://doi.org/10.1016/j.jbankfin.2011.03.006
BOYD, J. H.; HU, J.; JAGANNATHAN, R. The stock market's reaction to unemployment news: Why bad news is usually good for stocks. The Journal of Finance, 60(2):649-672. 2005. https://doi. org/10.1111/j.1540-6261.2005.00742.x

BURANELlO, R. Agronegócio: conceito. Enciclopédia jurídica da PUC-SP. Tomo: Direito Comercial. 1. ed. São Paulo: Pontifícia Universidade Católica de São Paulo. 2017.

CAMPBELL, J.; LO, A W.; MACKINLAY, A. The Econometric of Financial Markets. New Jersey: Princeton University Press. 1997.

DAVIS, J. H.; GOLDBERG, R. A. A. Concept of agribusiness. Division of Research, Graduate School of Business Administration, Harvard University, Boston, p. 136. 1957. https://doi.org/10.2307/1234228

DEVORE, J. L. Probabilidade e estatística para engenharia e ciências. Cengage Learning, $6^{\text {a }}$ edição. 2006.

DUARTE, P. C.; LAMOUNIER, W. M.; TAKAMATSU, R. T. Modelos econométricos para dados em painel: aspectos teóricos e exemplos de aplicação à pesquisa em contabilidade e finanças. In: Congresso USP de iniciação científica em contabilidade, São Paulo. Anais. São Paulo: FEA-USP. 2007. 
ELDOMIATY, T.; SAEED, Y; HAMMAM R.; ABOULSOUD, S. The associations between stock prices, inflation rates, interest rates are still persistent Empirical evidence from stock duration model. Journal of Economics, Finance and Administrative Science Vol. 25 No. 49, pp. 149-161. 2020. https:// doi.org/10.1108/JEFAS-10-2018-0105

FAMA, E. F. Efficient capital markets: a review of theory and empirical work. Journal of Finance, vol. $25, \mathrm{n}^{\circ}$ 2, p. 383-417. 1970. https://doi.org/10.2307/2325486

GUJARATI, D. N.; PORTER, D. C. Econometria básica. McGrawHill, 5a edição. 2011

HOULTHOUSEN, R.; VERRECHIA, R. The effect of informedness and consensus on price and trading volume behavior. The Accounting Review, 65, 191-208. 1990.

JR. GONÇALVES, W.; JR EID, W. Surpresas com relação à política monetária e o mercado de capitais: evidências do caso brasileiro. Revista de Economia Política, vol. 31, nº3, p. 435-454. 2011.

KHAN, K.; ZHAO, H.; ZHANG, H.; YANG, H.; SHAH, H.; JAHANGER, A. The Impact of COVID-19 Pandemic on Stock Markets: An Empirical Analysis of World Major Stock Indices. Journal of Asian Finance, Economics and Business Vol 7 No 7 (2020) 463 - 474. 2020. https://doi. org/10.13106/jafeb.2020.vol7.no7.463

KIM, O.; VERRECHIA, R. E. Trading Volume and Price Reactions to Public Announcements. Journal of Accounting Research, Vol. 29, No. 2 (Autumn, 1991), pp. 302-321. 1991.

KUTTNER, K. N. Monetary Policy Surprises and Interest Rates: Evidence from the Fed Fund Futures Market. Journal of Monetary Economics. vol. 47, $\mathrm{n}^{\mathrm{o}}$ 3, p. 523- 544. 2001.

MOREIRA, V. R. Gestão dos Riscos do Agronegócio no Contexto Cooperativista (Tese de Doutorado). Escola de Administração de Empresas da Fundação Getúlio Vargas, São Paulo, SP, Brasil. 2009.
NAKAO, S. H. Contabilidade financeira no agronegócio. $1^{\mathrm{a}}$ ed. São Paulo: Atlas. 2017.

PINDYCK, R.; RUBINFEL, D. Econometria: modelos e previsões. Elsevier. 2004.

ROBITAILLE, P.; ROUSH J. How do Brazilian yield spreads and stock prices respond a FOMC actions and U.S. macroeconomic data announcements? International Finance Discussion Papers 868, Federal Reserve Bank. 2006.

ROSS, S. A.; WESTERFIELD, R. W.; JAFFE, J. F. Administração financeira. $1^{a}$ ed. São Paulo: Atlas. 1995.

RUFINO, J. L. S. Origem e conceito do agronegócio. Informe Agropecuário, Belo Horizonte, v. 20, n. 199, p. 17-19. 1999.

SANTOS, J. O. Valuation - um guia prático. $2^{\mathrm{a}}$ ed. São Paulo: Saraiva. 2019.

USDA. Risks in agriculture. United States Department of Agriculture. Economic Research Service, 2020. Disponível em https://www.ers.usda.gov/topics/ farm-practices-management/risk-management/risk-in-agriculture. Acessado em 10/01/2021.

WONGSMAN, J. The response of global equity indexes a U.S. monetary policy announcements. Journal of International Money and Finance, vol. 28, $\mathrm{n}^{\mathrm{o}} 2$, p. 344- 365. 2009.

WOOLDRIDGE, J. M. Introdução a econometria: uma abordagem moderna. Cengage Learning. 2017. 